\title{
Application of security reference architecture to Big Data ecosystems in an industrial scenario
}

\author{
Julio Moreno ${ }^{1}$ (1) | Javier Gómez ${ }^{2}$ | Manuel A. Serrano ${ }^{3}$ | \\ Eduardo B. Fernandez ${ }^{4}$ | Eduardo Fernández-Medina ${ }^{1}$
}

${ }^{1}$ GSyA Research Group, University of Castilla-La Mancha, Ciudad Real, Spain

${ }^{2}$ Everis an NTT Data Company, Madrid, Spain

${ }^{3}$ Alarcos Research Group, University of Castilla-La Mancha, Ciudad Real, Spain

${ }^{4}$ Computer and Electrical Engineering and Computer Science, Florida Atlantic University, Boca Raton, Florida, USA

\section{Correspondence}

Julio Moreno, GSyA Research Group, University of Castilla-La Mancha, Ciudad Real, Spain.

Email: julio.moreno@uclm.es

\section{Funding information}

Consejería de Educación, Cultura y Deportes de la Dirección General de Universidades, Investigación e Innovación de la JCCM, Grant/Award Number: SBPLY-17-180501-000202; Ministerio de Economía y Competitividad, Grant/Award Number: RTI2018-094283-B-C31

\begin{abstract}
Summary
Big Data environments are typically very complex ecosystems; this means that implementing them is complicated. One possible technique with which to address this complexity is the use of abstraction. Reference architecture (RA) can be useful for an improved understanding of the main components of Big Data. Herein, we propose a security RA that includes the management of security concerns and provides the main elements of a Big Data ecosystem. Application of this architecture to real-world scenarios facilitates its refinement and improves its usefulness. In this article, we present a case study of a real-world Big Data ecosystem implemented in a banking environment. This ecosystem was developed by everis, an NTT company with which we collaborated for this study. To conduct this validation case study, a map was established between the elements of the Big Data ecosystem implemented and our proposal. Consequently, a series of valuable lessons that can improve both our architecture and the security of the Big Data environment were obtained. These include recommendations for a set of best practices such as the use of security patterns.
\end{abstract}

\section{K E Y W O R D S}

Big Data, case study, security patterns, security reference architecture

\section{1 | INTRODUCTION}

Big Data is rapidly transforming the manner in which organizations use their data. Over time, companies in every field are becoming increasingly aware of the importance of making use of all the data they collect. The data are essential for the daily operations of the companies and for improved decision-making based on the information extracted from them. ${ }^{1}$ Big Data adoption in businesses is showing increasing growth. According to a study of the Forbes magazine in 2018, ${ }^{2} 79 \%$ of the companies believe that Big Data will completely change the scenario of the industry in coming years. Moreover, the companies that fail to adopt a data analysis strategy based on Big Data will be in danger of losing market and their relevance. Consequently, Big Data ecosystems are being widely adopted in numerous domains such as transportation, ${ }^{3}$ healthcare, ${ }^{4}$ and manufacturing. ${ }^{5}$ However, the introduction of Big Data environment in an organization can cause significant changes in the business processes of the organization because it can lead to a change in the decision-making of the top management based on the insights generated by such an environment. 
Big Data ecosystem refers to a set of components that allow to store, process, visualize, and deliver useful information to target applications. Typically, these components are very complex and require collaboration to provide valuable insights to the company. ${ }^{6}$ This complexity further complicates the implementation of secure Big Data ecosystems. To address the problem of complexity, reference architectures (RAs) can be used for improved understanding of complex environments ${ }^{7}$ such as cloud systems. ${ }^{8}$

Furthermore, the inclusion of security elements in the RA facilitates improved understanding of such type of concepts and the identification of threats and security requirements for subsequent implementation of the system. Herein, the RA becomes a security RA (denoted "SecRA" in this article so as not to be confused with "software RA"). Consequently, we developed an SecRA proposal for Big Data ecosystems by considering the security issues and the inherent characteristics of Big Data ecosystems. ${ }^{9}$ This architecture prioritizes the use of security patterns to assist the implementation of security mechanisms while simultaneously controlling the threats to the system. Therefore, the use of SecRA allows an increased comprehension of both the Big Data ecosystems and their security through abstraction.

Our SecRA proposal is based on international standards, which were conducted in collaboration with the main companies in the sector. The application of this architecture to different real-world cases facilitates its improvement and increases its usefulness. In this article, we present the first approach for the validation of SecRA by comparing it with a real-world project. The project involves an existent scenario implemented by the everis company in its offices in Madrid, Spain, toward the creation of a Big Data ecosystem in a company in the banking sector.

The principal goal of this ecosystem is the integration of diverse data sources to create reports that assist the senior management in decision-making to optimize the sale of banking products and reducing risks to the bank. Toward this aim, the company has implemented an ad-hoc architecture that uses the Big Data technology. Typically, banking environment data is highly confidential and sensitive; therefore, the bank had imposed numerous security requirements on the Big Data ecosystem developers during implementation.

The main objective of this article is to prove the usefulness of our proposal by comparing it to a real-world environment that has been implemented by professionals in the given sector. Furthermore, from this objective, we can not only obtain a set of improvements that can be applied to our SecRA but also a collection of best practices and security improvements for the Big Data ecosystem that has been implemented in the banking environment. These best practices include a guide to implementing the security requirements of the given Big Data ecosystem by using well-known security patterns.

The remainder of this article is organized as follows. Section 2 explains the necessary background to understand this article. Section 3 describes the use case that we use to validate our proposal. Section 4 focuses on creating a mapping between the components of our proposal and the technologies implemented in the use case. Section 5 discusses the lessons learned from the validation including the improvements done to the SecRA and a set of best practices of security that the company can apply to its Big Data. Finally, Section 6 presents the conclusions and future work.

\section{2 | BACKGROUND}

An RA can be defined as an abstract software architecture that focuses on one or more domains and does not include implementation characteristics. To achieve this, an RA must be expressed using a high level of abstraction so that the architecture is reusable, extensible, and configurable for different scenarios. ${ }^{10}$ In the case of Big Data, different authors and organizations have proposed different RAs. However, among the proposals of the standardization organizations, the RA defined by the National Institute of Standards and Technology (NIST) has gained relevance in the industry; therefore, we define its proposal in greater detail. Furthermore, the ISO/IEC organization is currently working on the creation of an RA for Big Data under the ISO/IEC 20547-3 $3^{11}$ standard. This work is under development at time of the writing of this article; therefore, it is not possible to comment in detail about its content. In the following subsections, we describe the main proposals in detail.

\section{$2.1 \quad$ NIST RA for Big Data}

Over the past several years, NIST has defined an RA for Big Data that has received general approval from the industry and the scientific community. ${ }^{12}$ The latest version of this RA was released in June 2018, and it comprises numerous ideas and features to be able to create an architecture that abstracts any Big Data scenario. This set of features was elicited from the 
Big Data architecture proposals by leading companies in the industry such as Oracle and IBM. The RA is divided into five components that have different objectives and interact with each other. To deal with security problems, this architecture involves a security and privacy fabric that responds to the needs and solutions on this specific topic. In fact, there is a specific volume on privacy and security in Big Data. ${ }^{13}$ It highlights that the main security issues that a Big Data ecosystem can face are related to the following: (i) data confidentiality and privacy (this includes how to aggregate data without compromising their privacy), (ii) data provenance (how to ensure veracity and integrity of the data sources and the data already present in the Big Data ecosystem), (iii) monitoring of the Big Data ecosystem (to detect security breaches and incidents), and (iv) nefarious use of the environment by an internal attacker.

However, the NIST proposal cannot be considered as an SecRA because it does not address security as a primary requirement but as a fabric that is kept in the background. More specifically, security concerns are addressed from a holistic perspective rather than considering the security of every component of the Big Data ecosystem. From our point of view, this block-based representation is not sufficiently expressive. This type of specification has a considerably high level of abstraction, thereby providing little emphasis on the details of the sub-components and how they are connected. Therefore, this approach may not be adequately expressive to assist Big Data engineers in the design and implementation of such ecosystems. Although the NIST proposal involves these problems, it is a well-conceived and fairly comprehensive architecture; therefore, we used it as the basis to create our SecRA.

\section{2 | Our security RA for Big Data}

We created an SecRA based on the components proposed by the NIST that has the main objective of security improvement in Big Data systems. Our SecRA is aligned with the RA proposed by NIST; therefore, it can be easy to implement. Our architecture highlights the importance of implementing security solutions based on the concepts of the SecRA. This SecRA is described by using UML diagrams that endeavor to facilitate the implementation of secure Big Data. We decided on using UML diagrams because we came across very limited number of proposals, wherein the relationships between components and subcomponents were clearly displayed. Moreover, by using such diagrams, it is possible to apply different security patterns that are usually described as UML models. As aforementioned, this SecRA was published in detail in Reference 9.

Our SecRA emphasizes the definition of security requirements and solutions that are described in the first component of our architecture: the system orchestrator (SO). Subsequently, these artefacts are implemented in the remaining components of the SecRA. It is important that the security requirements are aligned with the goals and policies of both the organization and the Big Data environment. The security requirements can be satisfied through different security solutions that follow the organization's security policies and consider tackling threats and controlling vulnerabilities as their main objectives. To facilitate their implementation, our SecRA allows and encourages the use of security patterns. A security pattern is defined as a solution to a recurring problem. It describes how we can defend a threat or set of threats in a concise and reusable manner. ${ }^{14,15}$ Therefore, it can be said that the SO is the most abstract component of our architecture, and it would influence the implementation of the remaining components.

The data provider (DP) component creates an abstraction of the data sources considering their security metadata. It can be considered as a gateway between external data sources and the Big Data ecosystem. To this end, it establishes a series of interfaces that manage the security constraints of both the data sources and the Big Data ecosystem.

The next component of our architecture is the Big Data application provider (BDAP) that aims to meet the requirements established in the SO. To this end, the BDAP comprises the basic services that a Big Data ecosystem typically offers. They include the following five services: collection of the data that feed the analyses, preparation of data to improve the results by cleaning or structuring them, analysis techniques to obtain valuable information from data, visualization of the information obtained through charts or dashboards, and access control to data. Not all Big Data ecosystems are required to provide all these services; there are some optional services such as the preparation or visualization of results, which depending on the requirements of the ecosystem to be developed, may not be necessary. These services are implemented at the hardware level in the following component.

The Big Data framework provider (BDFP) component implements the services offered by the BDAP. For this purpose, it usually comprises one or more clusters that are composed of nodes. In addition to hardware infrastructure, this component provides storage, processing, and other services such as communication and resource management. Currently, several companies (especially small and medium) outsource this part of the architecture, on which they build their Big Data ecosystem, to a commercial cloud solution on a contract basis. 
This simplifies the development of the Big Data ecosystem and reduces the costs of purchasing the necessary hardware.

Finally, the last component of our architecture is the data consumer (DC). The main aim of this component is to provide the information generated by the Big Data ecosystem, thereby acting as a connection between the environment and the end user of the data. The end user can also indicate another connected system apart from a human user.

Figure 1 illustrates our complete SecRA for Big Data. The figure shows how the components and elements of our architecture relate to each other. Furthermore, it illustrates how the security solutions defined in the SO are implemented in the remaining components of the ecosystem. Regarding this, the aforementioned security patterns hold considerable importance because their objective is to facilitate the implementation of the security solutions. The following section explains the developed Big Data ecosystem that serves as a case study to validate our SecRA proposal.

\subsection{Other proposals of RAs for Big Data}

Among the other proposals of RAs for Big Data, Demchenko et $\mathrm{al}^{6}$ proposed a Big Data framework architecture that establishes the data life-cycle of a Big Data ecosystem. Similar to the NIST approach, they use a block diagram representation but with more details in the relationships between the components of the architecture. Furthermore, in another similarity to the NIST proposal, they approach security as an isolated feature-not really connected to the other components. Nevertheless, they provide some interesting contributions in terms of explaining the main components of Big Data ecosystems, wherein security should be emphasized. To validate their proposal, they illustrate an example of how a system using Big Data can be deployed for a university.

Another research on this topic is Reference 16, where the authors proposed a complete architecture in terms of the relationships between the components. Toward this aim, they conducted a complete study of the different proposals in this field and created their own RA based on the main components of a Big Data ecosystem. To validate their proposal, they compared their architecture to the Big Data ecosystem implementations of well-known companies, for example, LinkedIn, Facebook. Although theirs is a very comprehensive and complete proposal, they do not consider security as a key aspect in the Big Data ecosystem because they do not address it in their proposal.

In Reference 17, the authors propose a software RA for semantic-aware Big Data ecosystems, it follows the lambda architecture principles, to which the authors add a semantic layer. They validate their proposal by adopting their architecture in three projects. They provide an interesting approach; however, their proposal is more focused on the data life-cycle in a Big Data ecosystem. They do not consider security issues as an objective. The lambda architecture, that is, $\lambda$-architecture, is based on dividing the Big Data ecosystem into different layers depending on how data are processed and managed: in real-time (speed layer), making queries on stored data (batch layer), and showing the results of the queries made (the serving layer). ${ }^{18}$ This type of architecture is widely used in the implementation of Big Data systems. ${ }^{19,20}$

Klein et $\mathrm{al}^{21}$ proposed a very specific RA for Big Data that focused in addressing typical national defense requirements. Their architecture is considerably similar to the one proposed by NIST. They include a tutorial demonstrating the usage of their architecture to create concrete Big Data solutions. Similar to the NIST proposal, they have a dedicated security module to address privacy and security issues-they treat security as an isolated feature that does not follow the security-by-design perspective. Nonetheless, this module highlights some interesting features regarding the security aspects that should be considered in Big Data ecosystems, for example, controlling user access to data and intrusion detection and prevention.

Mistrik et $\mathrm{al}^{22}$ proposed a software architecture for Big Data that covers the first phases of the life-cycle of a Big Data ecosystem-from design to implementation. However, similar to other proposals, this proposal contemplates security as a complement, and not as a crucial feature for successful implementation. However, they provide an interesting perspective in terms of creating an environment, wherein users are aware of the need to preserve data privacy.

In Reference 23, the authors propose a reference model for industrial Big Data that has components similar to the ones defined in the NIST proposal. They consider security-more specifically, cybersecurity, as an important aspect of such Big Data environments. However, they only address it as a set of steps during the development of a Big Data ecosystem, and not as an integral requirement while implementing a component.

Among the industry proposals, the ones of the main companies comprise an architecture based on their own technological stack, for example, Microsoft, IBM, and Oracle. None of these proposals can be considered as an SecRA because they do not highlight the importance of security. 


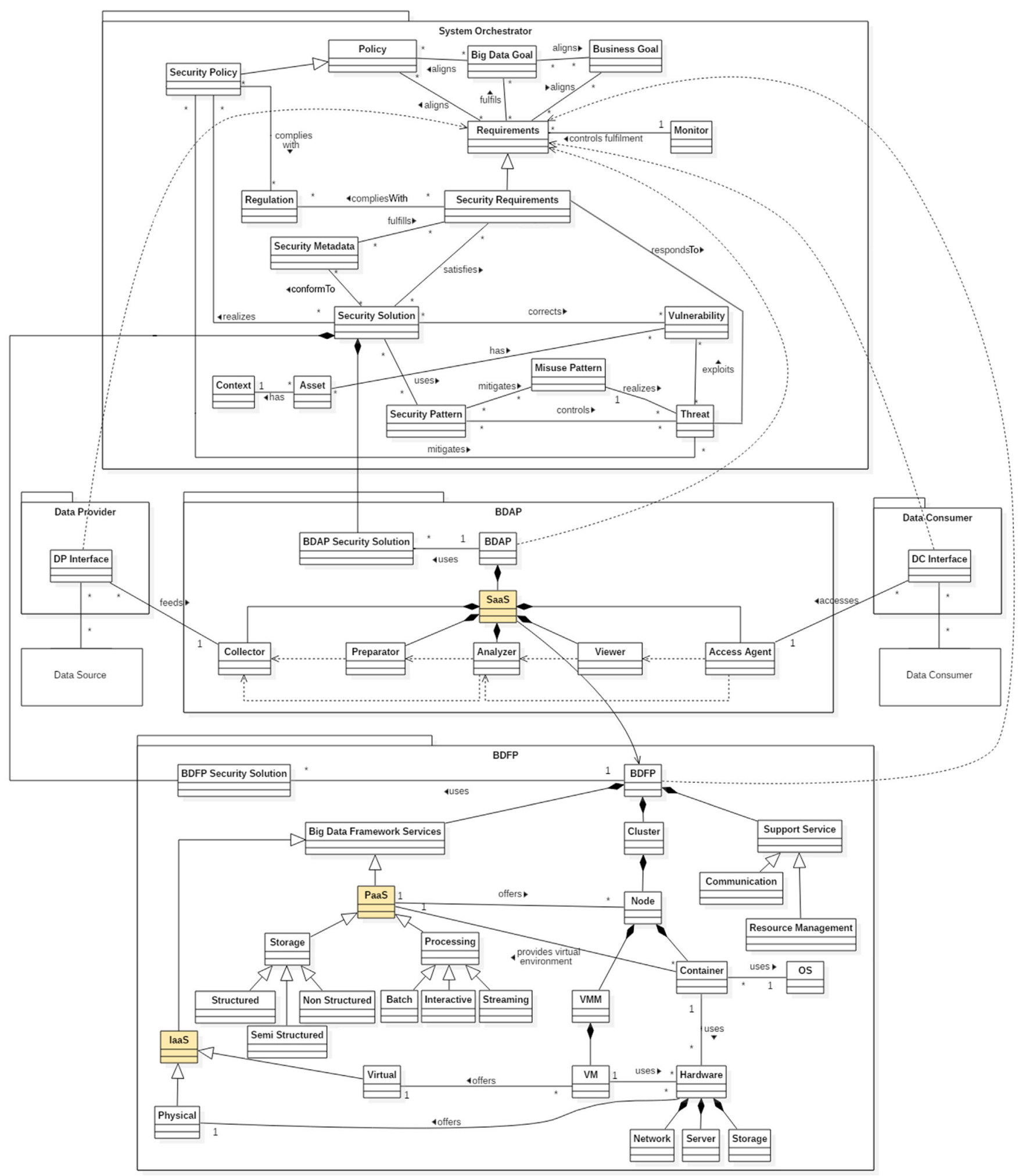

F I G U R E 1 Proposed SecRA for Big Data ecosystems [Colour figure can be viewed at wileyonlinelibrary.com] 
T A B L E 1 Comparison between RA proposals for Big Data ecosystems

\begin{tabular}{|c|c|c|c|c|}
\hline RA Proposal & $\begin{array}{l}\text { Security } \\
\text { conside-ration }\end{array}$ & $\begin{array}{l}\text { Connection } \\
\text { between components }\end{array}$ & $\begin{array}{l}\text { Abstraction } \\
\text { level }\end{array}$ & $\begin{array}{l}\text { Use of } \\
\text { security patterns }\end{array}$ \\
\hline $\mathrm{NIST}^{12}$ & High & Low & High & No \\
\hline Demchenko et $\mathrm{al}^{6}$ & Medium & Medium & Medium & No \\
\hline Pääkkönen and Pakkala ${ }^{16}$ & Low & High & Medium & No \\
\hline Nadal et $\mathrm{al}^{17}$ & Low & Medium & Low & No \\
\hline Klein et $\mathrm{al}^{21}$ & Medium & Medium & Low & No \\
\hline Mistrik et $\mathrm{al}^{22}$ & Medium & High & Low & No \\
\hline Zhan et $\mathrm{al}^{23}$ & Medium & Low & High & No \\
\hline Our SecRA proposal & High & High & Medium & Yes \\
\hline
\end{tabular}

Abbreviations: RA, reference architectures; SecRA, security RA.

In a summary of this section, Table 1 provides a comparative overview of the proposals analyzed so far, including ours. This comparison considers the main characteristics of our proposal to highlight its primary differences with the other RA proposals.

In a conclusion of this section, it can be observed that most of the RA proposals for Big Data ecosystems do not have security as a main consideration; they consider it as an extra module that is not integrated with the remaining functionalities of the environment. However, our architecture addresses security at the onset of the development of a Big Data ecosystem by incorporating security patterns to aid the implementation of security requirements.

\section{3 | CASE STUDY: BIG DATA ECOSYSTEM FOR A BANK APPLICATION}

In Section 3.1, we describe the context and the research method used to conduct the case study. Subsequently, in Section 3.2, we explain the purpose and the main characteristics of the developed Big Data ecosystem.

\section{1 | Context and research method}

Everis, an NTT DATA Company, is a multinational consulting firm that offers business solutions, strategy, digital transformation, development and maintenance of technological applications, and outsourcing. It operates in 15 countries, and employs a total of 17000 professionals. It has a turnover of 816 million euros. Moreover, NTT DATA is the 10th largest ICT services company in the world and is a part of the NTT Group.

Everis' solution to implement the Big Data ecosystem serves as the basis for comparison with our SecRA proposal. Owing to everis' confidentiality agreement with the bank, certain specific details of the implementation cannot be disclosed. The action research method was followed to conduct the case study. ${ }^{24}$ This well-known method is based on generating a benefit for the research "client" (here, everis) and simultaneously generating relevant data on "research knowledge." 25 This results in a win-win situation wherein both parties benefit from the application of the method. Figure 2 illustrates the roles involved in this case study and how they relate to each other. To collect data about the case study, a number of interviews were conducted with the developers and architects of the implemented Big Data ecosystem.

As shown in Figure 2, the action research method follows an iterative approach in different steps:

- In the first step, planning, the objects of the study are defined, and the method to conduct the case study is decided. In our case, the objects of study comprised the Big Data banking ecosystem and the SecRA. Furthermore, it was decided to conduct the case study by comparing the components of the objects of study.

- Next, the protocol to conduct the case study is instantiated. In our case, a mapping was established between the components and the elements. 


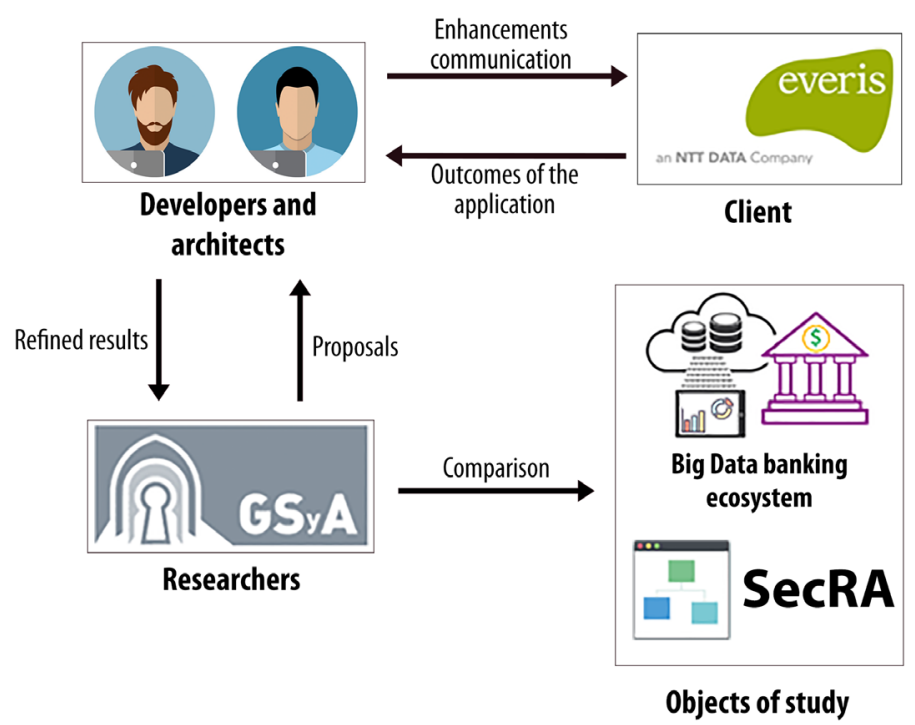

F I G U R E 2 Application of the action research method to our scenario [Colour figure can be viewed at wileyonlinelibrary.com]

- In the subsequent step, the results are observed and the data are collected and documented. In our case, the results are presented in Section 4.

- Finally, in the last step, the results are analyzed to obtain an analysis useful for both parties. This step may lead to the conclusion that another iteration of the case study is required, and it can be conducted by changing certain values to obtain different results. In our case, a series of lessons were learned-they would facilitate the improvement of our SecRA and the Big Data banking ecosystem.

Validation of an SecRA is not trivial; it requires a number of iterations that allow the refinement of proposal until it can be considered adequately mature. To this end, each case study must be conducted in a different scenario, thereby analyzing a different Big Data environment, which would demonstrate that the SecRA is sufficiently complete to cover any context. In this article, we present the first case study and its conclusions. Figure 3 illustrates the process of creation and validation of our proposal and its comparison with the Big Data ecosystem developed by everis.

\section{2 | Big Data banking ecosystem}

The main goal of the developed Big Data ecosystem is the generation of reports that help management in decision-making such as implementing measures to optimize the sales of banking products. Furthermore, profiling operations are conducted on the bank customers to reduce the risks to the bank. To achieve these objectives, a variety of input data sources such as navigation events generated in the web application, customer information (from know your client processes and client databases from the company), anti-money laundering processes, and other systems of the bank can be utilized.

Furthermore, a lambda architecture with two layers was implemented. The speed layer processes and analyzes the streaming data with characteristics that are close to real-time. The batch layer stores the necessary data for on-demand analysis to generate reports or views for the bank's top management. Figure 4 presents the system architecture and the components that comprise it.

In the speed layer, the data are input into the ecosystem through a messaging broker that has an interface for each data source and functionality of the received data. Subsequently, the processing layer reads and processes the data according to the defined requirements to generate useful information for consumption. Here, micro-batch processing that simulates an analysis of the data in real-time is employed. The results are stored in an NoSQL storage (Real Time Views in Figure 4). This storage is modeled for the ease of understanding for consumption and use for queries. Consequently, the access to information is optimized to reduce access latency. Finally, data consumption happens through the use of query services or tools.

In the batch layer, data are input from two sources: (i) data processed by the speed layer and (ii) data obtained from different databases or bank systems. These data are processed using four layers organized according to the status of the 
F I G U R E 3 Process to create and validate the SecRA compared to the banking Big Data ecosystem [Colour figure can be viewed at wileyonlinelibrary.com]

\section{SecRA}

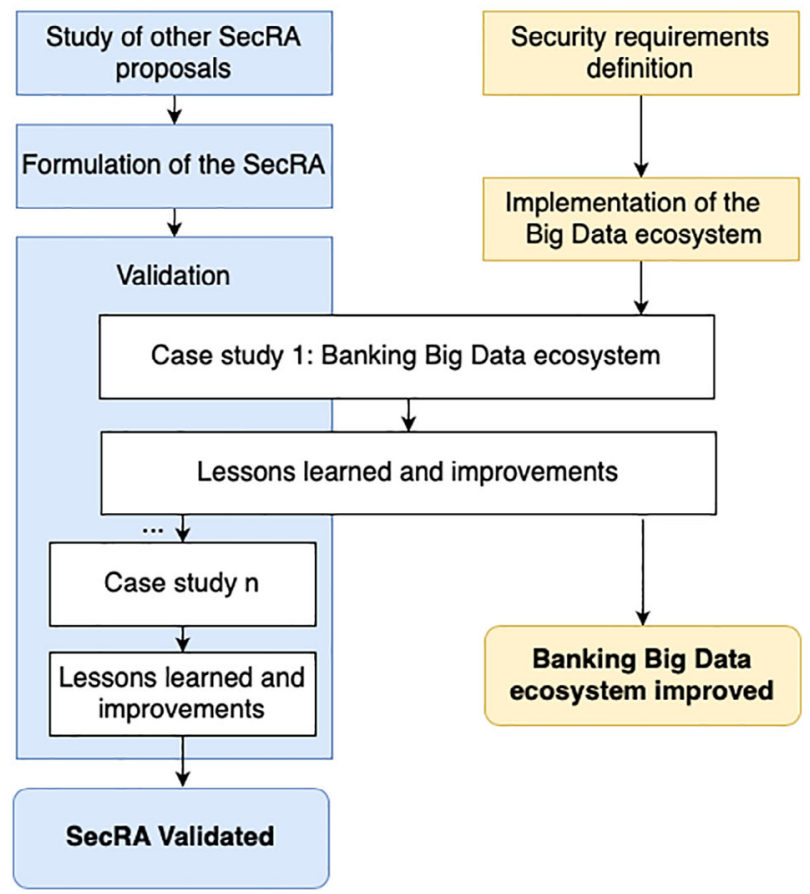

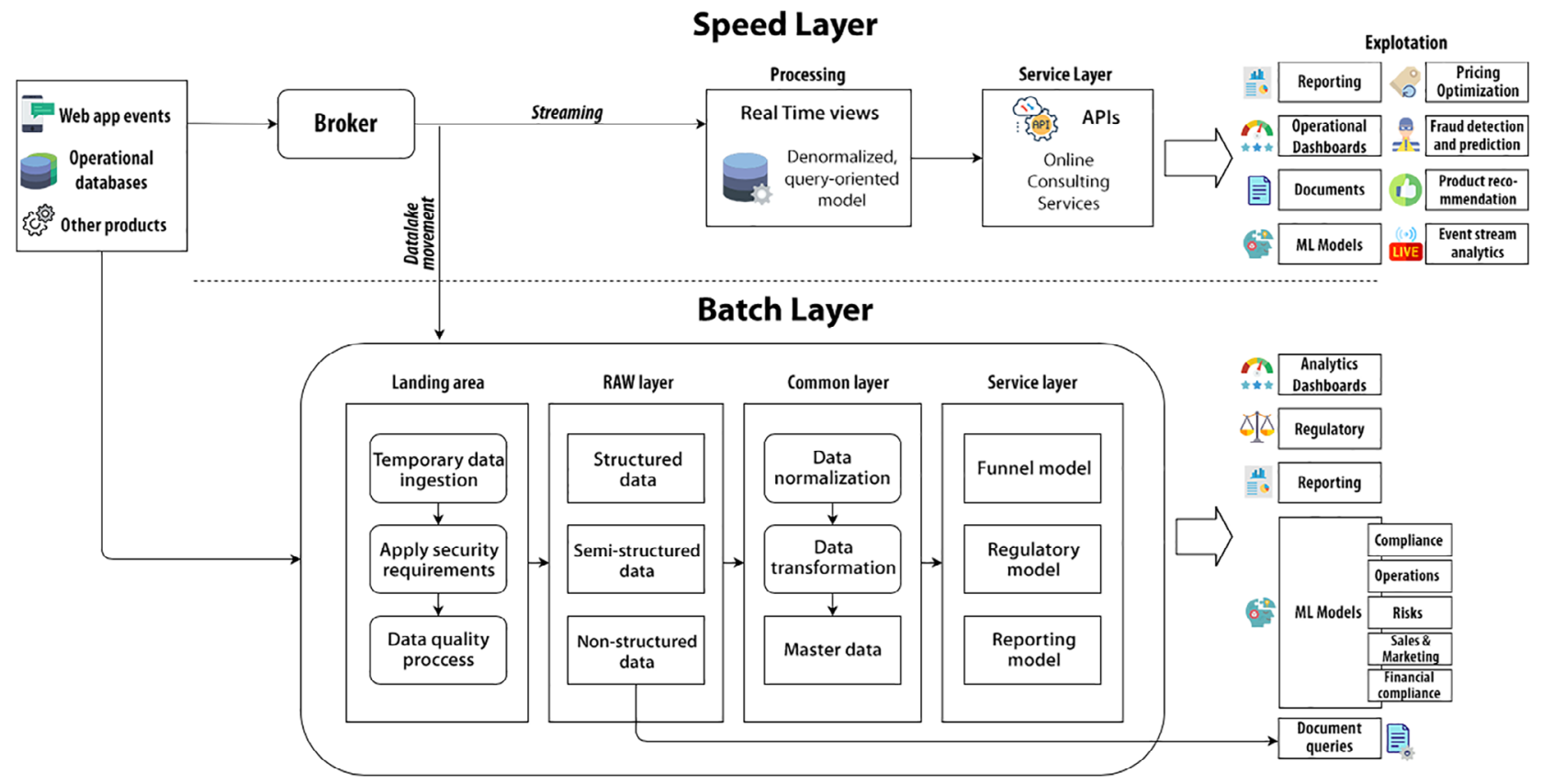

F I G U R E 4 Architecture of the case study [Colour figure can be viewed at wileyonlinelibrary.com]

data or its subsequent use. These layers can be considered as the life cycle of the data in the ecosystem, and they are explained as follows.

- Landing area: This is a temporary space wherein raw unprocessed data are received for the purpose of applying procedures related to the regulations affecting the system, for example, anonymization or pseudo-anonymization of data. This layer comprises three steps: (i) data are input on a massive scale without performing quality controls, (ii) security and data protection regulatory requirements such as general data protection regulation are applied (depending on the 
sensitivity of the data, different controls will be applied), and (iii) processes are applied to evaluate and establish rules on the metadata of the data received, for example, the file format or the compression applied. Data in this layer are raw and not anonymous; therefore, only technical and very specific users have access to it. For improved protection of the data in this layer, it is kept separate from the remaining data.

- Raw layer: This is a massive data storage space wherein structured, semi-structured, and nonstructured data are stored after applying the regulatory processes of the previous phase. These data are considered to be raw because no mechanism is applied to prepare them for further analysis. The data in this layer are used for operational functions, advanced analytics, and successive processing of the remaining layers. This layer includes metadata on the origin, functionality, and entity of the data source.

- Common layer: This layer is dedicated to the construction of business entities such as customers, contracts, and products. These entities aim to provide a unique and centralized functional vision. This layer can be considered as the data preparation phase. It comprises three steps: (i) cleaning and normalization of the data to establish a single standard of coding and rules, (ii) transformation of the data with the application of business logic, and (iii) generation of master data based on requirements. The master data is used to employ advanced analytical techniques and for the generation of artefacts to be used in the service layer.

- Service layer: This layer is dedicated to handle business needs. It hosts the necessary entities for the monitoring and decision-making of the company. It is divided into different departmental functions, thereby allowing analysis by department or as a global vision of the business. This layer prepares the data entities to generate reports and information consumption mechanisms.

For the implementation of the proposed architecture, Azure's cloud solution was chosen to simplify the development and make the necessary hardware management transparent. More specifically, the second-generation Azure Data Lake Storage solution was chosen. Snowflake was used to facilitate the use of the data lake by adding a layer to use it as a data warehouse. Apache Spark was used as the main engine for data analysis. The analytical capabilities are not limited to Spark—other technologies are used to complement its features, for example, Delta Lake to provide transactional support between the data, Azure Databricks to generate a workspace use of Spark in a more intuitive manner, and Azure Machine Learning Studio to easily create and deploy predictive analytics solutions.

The relationship between all these technologies is not simple. To solve this problem, Azure Data Factory was used to integrate and orchestrate the different processes of the Big Data ecosystem and Apache Kafka to facilitate the communication between the applications. To control the access to the system and the authentication of the roles and users, the following tools provided by Azure were used: Key Vault to protect the keys and secrets used by the applications and Active Directory to manage the identities of the users of the system. Finally, the generation of reports and visualization of the information obtained from the application of the analytical processes was performed with the Power BI tool. Figure 5 illustrates the technological instantiation of the architecture.

The bank client laid down a set of security requirements that must be met by the developed ecosystem. These requirements emphasized the need to ensure data confidentiality owing to the high sensitivity of the data and to comply with the legal regulations of the banking context. These requirements were defined by the client after an analysis of the vulnerabilities, threats, and risks that could affect the Big Data ecosystem being developed. These restrictions are especially rigorous owing to the context of the ecosystem, wherein data are treated with a very high level of sensitivity. To satisfy these requirements, a table of correspondence was created between the security requirements and the technologies used. The objective of this table is to focus on the cases in which the technology has certain configuration options to address each security requirement or to identify if there is a requirement that must be implemented in another manner (through software solutions or delegated to other components). Table 2 lists the security requirements and how they are implemented in different technologies.

As shown in the table, majority of the security requirements are relatively easy to apply because the technology supports them. However, there are cases wherein the tool does not support the requirement but delegates its compliance to another related tool for example, Delta Lake is deployed as a layer above the Azure Data Lake; therefore, it delegates most of the security needs to Azure Data Lake ("DL" in Table 2), Apache Spark uses Databricks workspace and its security ("DB" in Table 2).

Some requirements do not apply in some cases because they do not affect the technology, for example, Active Directory does not store any type of data at rest. Finally, in the case of managing the use of highly sensitive data that neither should be stored nor accessed by all users is delegated to the landing layer ("L" in Table 2). As explained above, this layer 


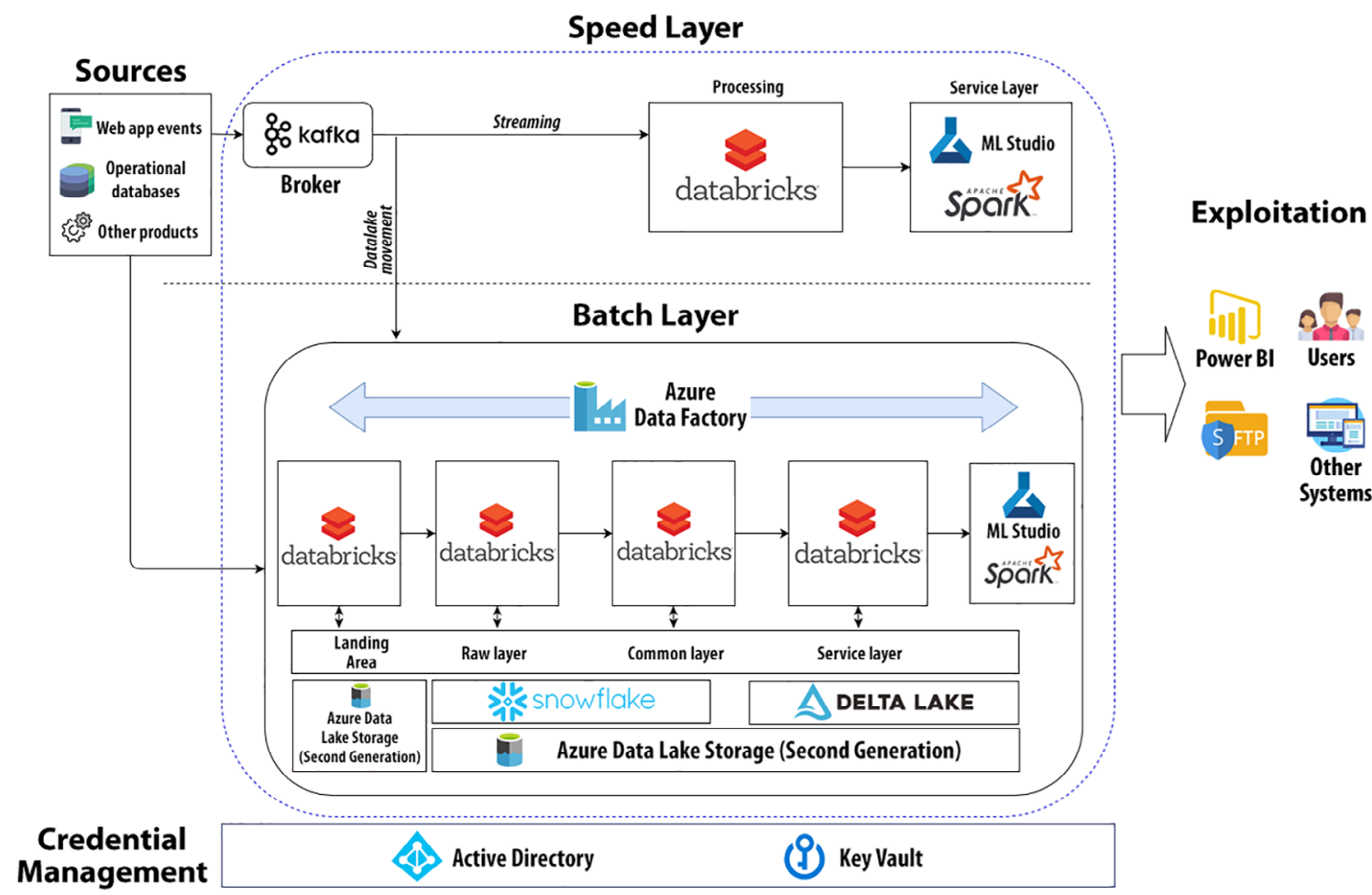

F I G U R E 5 Technological instantiation [Colour figure can be viewed at wileyonlinelibrary.com]

excludes data that should not be added owing to their high sensitivity or encrypts data whose content is accessible only to certain users using pseudo-anonymization techniques. In conclusion, this section defined a real-world system that was well implemented with security requirements and well resolved without following a specific methodology but based on the experience (know-how) of the developers, which allows an optimal selection of technologies that best suit the required scenario.

The following section discusses a comparison between the concepts of our SecRA and the actual Big Data ecosystem implementation for checking the similarities and studying the differences between the two.

\section{4 | MAPPING BETWEEN SECRA AND BIG DATA BANKING ECOSYSTEM}

This section presents an in-depth discussion of the differences and similarities between our SecRA proposal and the case study explained in the previous section. To this end, componentwise mapping is carried out for our architecture and it is made clear how each element is implemented in the Big Data banking project. It is important to highlight that some elements of our architecture can be optional in the Big Data ecosystem. This is owing to the fact that our architecture aims to cover all scenarios; therefore, some elements may not necessary in some cases depending on the requirements of the system to be developed.

We first focus on the BDFP component of our architecture. As aforementioned, the main objective of this component is to support the services offered by the Big Data ecosystem and provide support functions for these services. The case study reveals that everis has decided to implement the Big Data ecosystem on an Azure cloud solution, specifically a second-generation data lake storage. Our SecRA covers this scenario because the outsourcing of hardware resources greatly facilitates its management. It is interesting to understand how this storage system is managed, because in the case study, it was divided into several layers depending on the state of the data and who can make use of it. In our SecRA, we did not perform storage segmentation; however, we covered its functionality through the application of access controls for the roles in the company.

Furthermore, our architecture does not make an obvious distinction between the different forms of processing (batch and streaming in the case study) but contemplates the different forms of processing that are typical of Big Data ecosystems. 


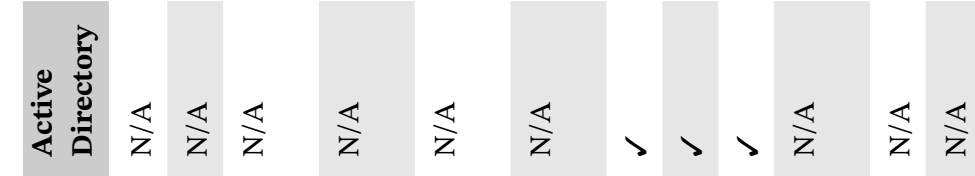

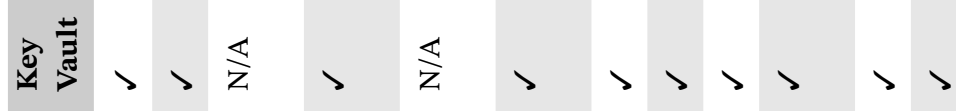

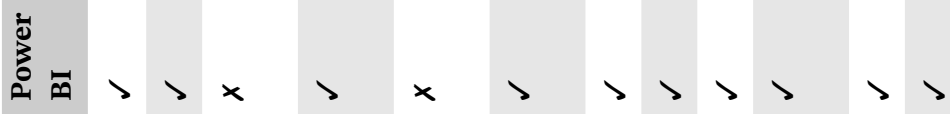

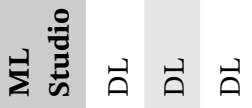

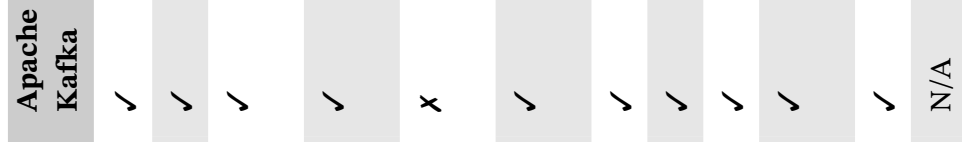

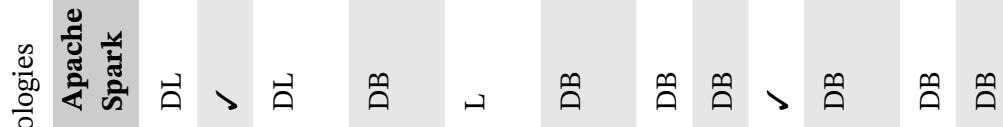

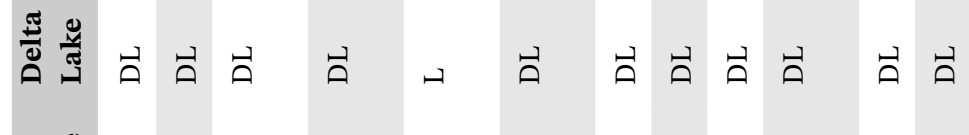

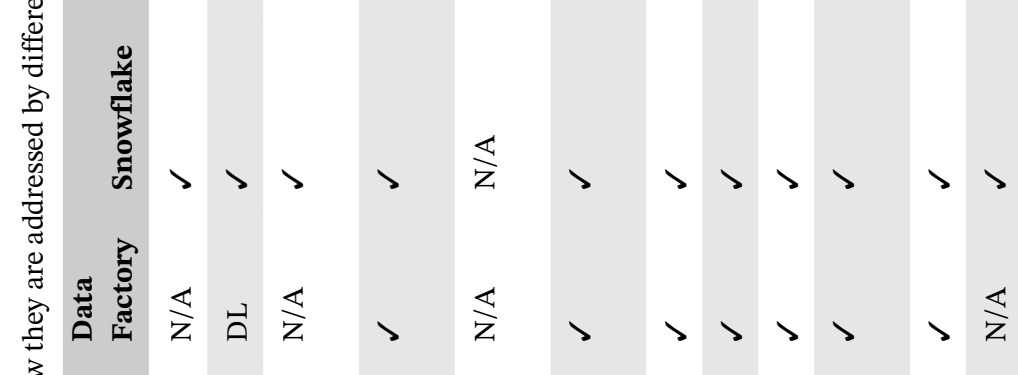

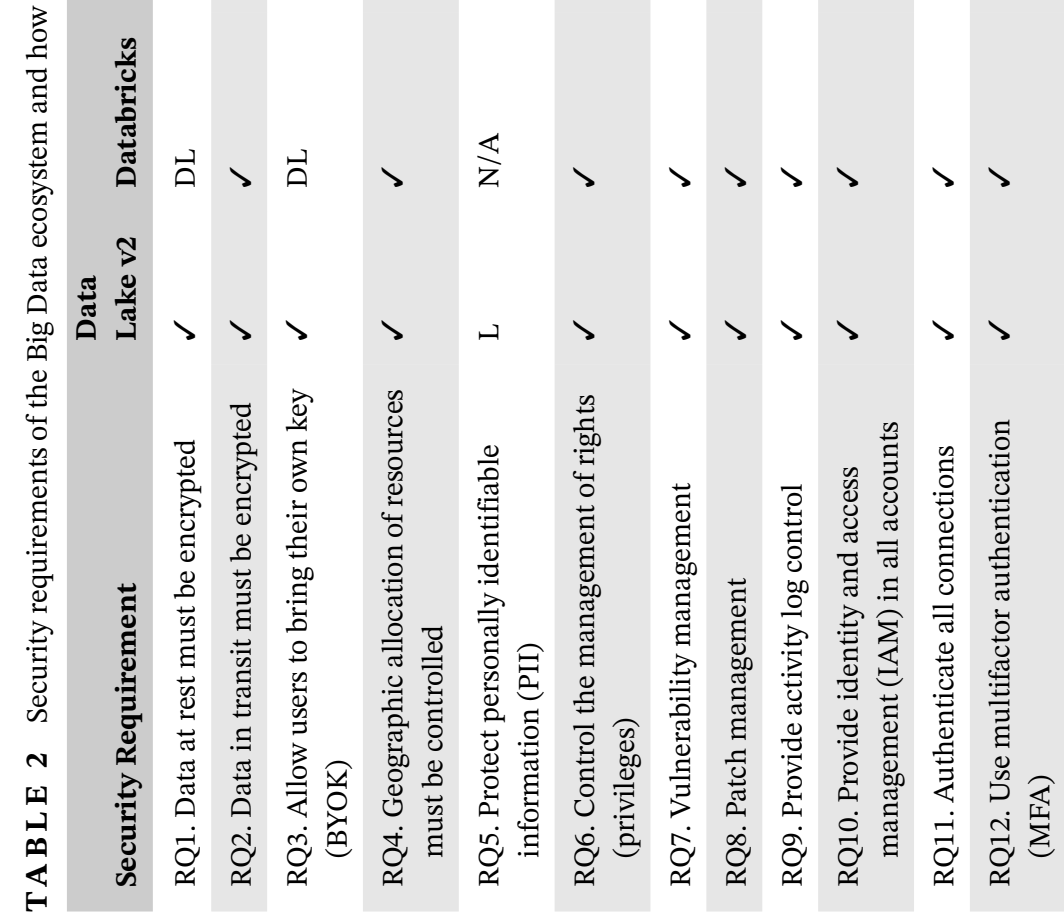


TA B L E 3 Mapping between the BDFP elements and the tools used in the case study

T A B L E 4 Mapping between the BDAP elements and the tools used in the case study

\begin{tabular}{|ll|}
\hline SecRA (BDFP) & $\begin{array}{l}\text { Big Data } \\
\text { Banking Ecosystem }\end{array}$ \\
\hline Cluster & $\begin{array}{c}\text { Azure Data Lake Storage (Second } \\
\text { Generation) }\end{array}$ \\
\hline Big Data Framework Services & \\
\hline PaaS & Databricks \\
\hline Storage & Delta Lake and Snowflake \\
\hline Processing & Apache Spark \\
\hline IaaS & Azure Data Lake Storage (Second \\
\hline Support Services & Generation) \\
\hline Communication & Apache Kafka \\
\hline Resource Management & Azure Monitor \\
\hline BDFP Security Solutions & Implemented in each tool (see Table 2) \\
\hline
\end{tabular}

Abbreviations: BDFP, Big Data framework provider; SecRA, security reference architecture.

\begin{tabular}{|ll|}
\hline SecRA (BDAP) & Big Data Banking Ecosystem \\
\hline Collector & $\begin{array}{c}\text { Landing area (Azure Data Lake Storage (Second } \\
\text { Generation) and Databricks) }\end{array}$ \\
\hline Preparator & Apache Spark scripts \\
\hline Analyzer & Apache Spark scripts and ML Studio \\
\hline Viewer & PowerBI \\
\hline Access Agent & Azure Active Directory and Key Vault \\
\hline BDAP Security Solutions & Implemented in each tool (see Table 2) \\
\hline
\end{tabular}

Abbreviation: BDAP, Big Data application provider.

Table 3 lists the elements of BDFP and the technology used for their implementation in the case study. Overall, it can be concluded that our SecRA fairly and completely covers all the artefacts implemented by the case study with the exception of the support service for orchestration that is implemented in the case study by using Apache Kafka.

The next component, BDAP, integrates the different services that a Big Data ecosystem offers-it can be considered as the Software as a Service layer. In this case, the Big Data ecosystem implements all the services planned by the SecRA. It is interesting the case of the data collection, because the Big Data bank project implements a first auxiliary and temporary layer (landing area) that is separate from the main data lake. This layer contains data that are inaccessible, and they are kept in a temporary storage until security policies are applied to transform them to be usable. Therefore, no specific technology, but the landing area (implemented using second-generation Azure Data Lake Storage and managed using Databricks) that ingests data into the Big Data ecosystem is used to implement this element of the SecRA.

No specific tool was used for data preparation; however, the role of the data scientist can implement scripts in Apache Spark to improve the quality of data prior to analysis. This analysis can be performed by implementing algorithms in Apache Spark using Python, Java, and Scala; alternatively, the software MLStudio can be used for this. During data visualization, the PowerBI tool is used to generate reports and track KPIs to create dashboards. The last service contemplated by the SecRA is access control to the remaining Big Data ecosystem services; in the case study, this service was implemented using the tools provided by Azure: Active Directory and Key Vault. Table 4 lists the elements of the BDAP component and the technology used for their implementation in the case study.

In our architecture, the DP component serves as a gateway to the Big Data ecosystem through a series of interfaces with the aim of applying the security policies of both the Big Data and the original data sources. In the case study, this function is blurred and included within the landing area that is a part of the Big Data ecosystem. This difference is important because 
T A B L E 5 Mapping between the elements of the DP, DC, and SO, and the tools used by the case study

\begin{tabular}{lll} 
SecRA Component & SecRA Element & $\begin{array}{l}\text { Big Data } \\
\text { Banking Ecosystem }\end{array}$ \\
\hline DP & DP Interfaces & Landing area and Azure Data Factory \\
\hline DC & DC Interfaces & Access control to the ecosystem and SFTP \\
\hline SO & Security Policy & Defined by the client \\
\hline SO & Policy & Defined by the client \\
\hline SO & Business Goal & Defined by the client \\
\hline SO & Big Data Goal & "To obtain benefits from the bank available data sources" \\
\hline SO & Requirements & Defined by the client \\
\hline SO & Security Requirements & See Table 2 \\
\hline SO & Regulation & Defined by the client \\
\hline SO & Monitor & Not used in the case study \\
\hline SO & Security Metadata & Not present in the case study \\
\hline SO & Security Solution & See Table 2 \\
\hline SO & Vulnerability & Not present in the case study \\
\hline SO & Threat & Not present in the case study \\
\hline SO & Security Pattern & Not used in the case study \\
\hline SO & Misuse Pattern & Not used in the case study \\
\hline SO & Context & Defined by the client \\
\hline & See Figures 4 and 5 \\
\hline
\end{tabular}

Abbreviations: DC, data consumer; DP, data provider; SO, system orchestrator; SecRA, security reference architecture; SFTP, secure file transfer protocol.

our architecture recommends that these verifications be conducted before and outside of the ecosystem to improve data security and privacy. However, the case study contemplates how to connect the data sources with the Big Data ecosystem via the extract, transform, load process; more specifically, by using the Azure Data Factory. The DC component at the end of the Big Data ecosystem aims to connect the system to end users. In the case study, this connection is realized through two different methods: through access control to the visualization of the generated reports and dashboards and file sharing using the secure file transfer protocol.

Finally, SO, the last component of our architecture is defined during the first phases of the development of the Big Data ecosystem. As aforementioned, the objective of this component is the realization of different security requirements and the security solutions that satisfy them. The present case study is an already implemented Big Data system; therefore, the first phases of development are reduced to a set of security requirements and the main objective of the Big Data banking system. Elicitation of these security requirements was performed by the client, for which they considered the main legal regulations that could affect the system and the bank's security policies.

In this case, a considerable difference exists between our SO component and everis' approach because in our SecRA, while defining the security requirements, we considered the different vulnerabilities and threats of the ecosystem to be developed. Moreover, in our architecture, the security and the misuse patterns have an important role because they facilitate the implementation of security solutions. Moreover, the SecRA has a specific element: monitor that observes the behavior of the system to check that it remains aligned with its requirements. Table 5 summarizes the main elements of the aforementioned three components and how they relate to the case study.

Figure 6 summarizes the relation of the technologies implemented in the banking Big Data ecosystem to each component of our architecture. It can be seen from this figure that the SO component does not contain any element can be remarked. This is because of the fact that the requirements usually defined and monitored in this component were defined by the bank client; therefore, everis was not required to define these artefacts; however, it had to define how to fulfill these requirements. Furthermore, BDFP may seem like a reduced version compared to our architecture. This is because 
F I G U R E 6 Summary of the main SecRA components and technologies used in the case study [Colour figure can be viewed at wileyonlinelibrary.com]

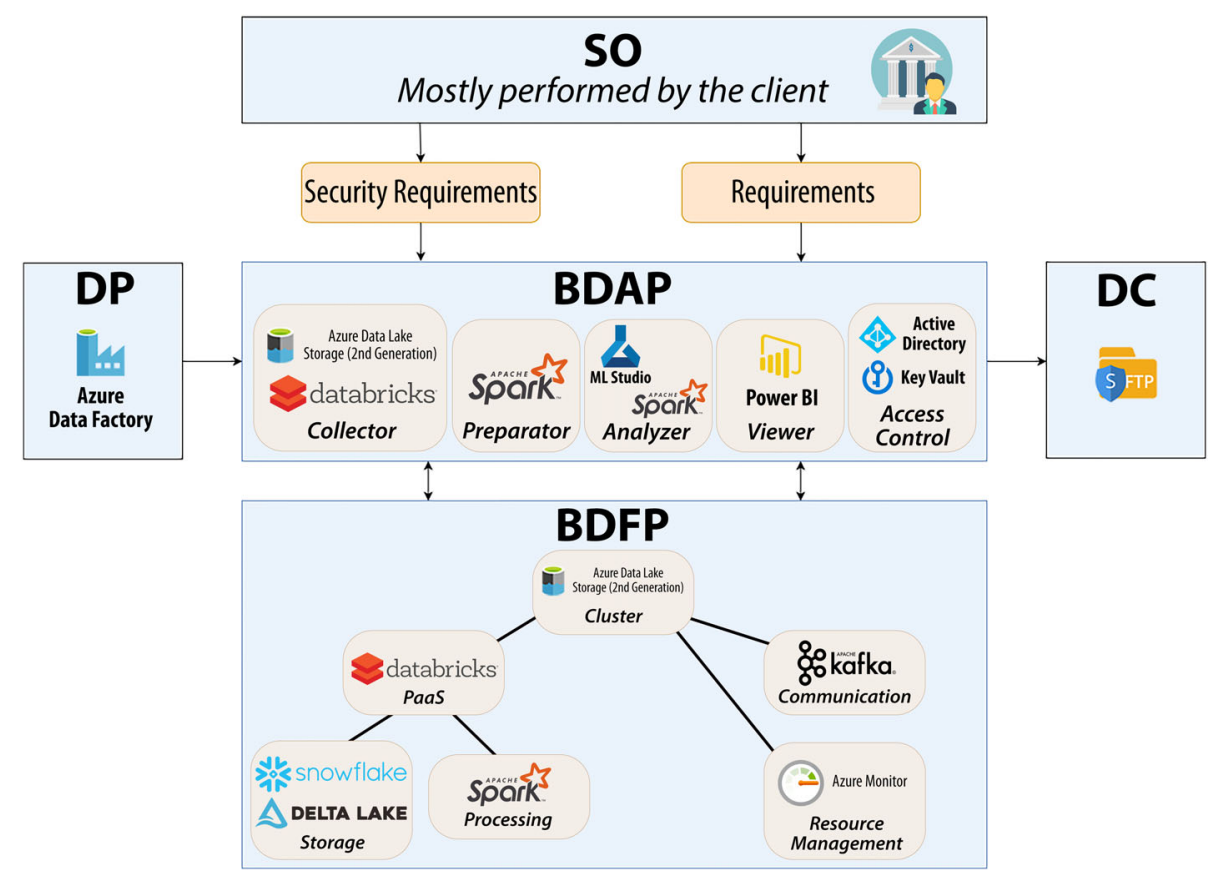

our SecRA considers all possible scenarios of implementation of a Big Data ecosystem, whereas in a normal scenario, it is not necessary to use all the elements. In this case study, the Azure platform that reduced the complexity of the developed ecosystem was used.

It is important to reiterate that one of the main objectives of SecRA is to cover any type of Big Data ecosystem regardless of its context or requirements. This means that it is possible to map our SecRA to any scenario, wherein a Big Data ecosystem is implemented because all elements of such an ecosystem are present in our SecRA proposal.

Once the mapping between the case study and the SecRA was completed, a series of lessons were learned and conclusions were obtained that were valuable both for the bank and us. They are presented in the following section.

\section{5 | LESSONS LEARNED}

After comparing the elements of the SecRA and those implemented in a real-world case study, we obtained a set of conclusions that can be used to improve both our architecture and the case study. In addition, we obtained a series of general conclusions that can be useful for the effort involved in the implementation of any type of project for the implementation of a Big Data ecosystem.

During the experiment, we realized the effort involved in the implementation of a complex Big Data ecosystem, wherein multiple technologies connected to each other work concurrently. To mitigate this complexity problem, it is necessary to apply considerable effort in the planning phases: both analysis and design of the Big Data project. Furthermore, some classic security requirements are complicated to implement in such type of ecosystems, for example, treatment of the granularity of the data for access control that becomes increasingly complex owing to the amount of data required to be handled. Another problem encountered in the project was the difficulty of appropriately implementing legacy data in distributed environments with multiple nodes having different configurations and tools executing different tasks.

\section{1 | SecRA improvements}

Practically, all elements of our SecRA were represented in the case study; this proves the validity of SecRA in terms of adapting to any type of Big Data scenario. However, we identified certain details that could improve our proposal; all of them belong to the BDFP component. This offered us an improved understanding of how a Big Data ecosystem can be implemented in a real-world scenario. Furthermore, this knowledge enables us to refine our SecRA to ensure that it covers all possible scenarios. Moreover, we discovered that our architecture did not offer service support in an evident 


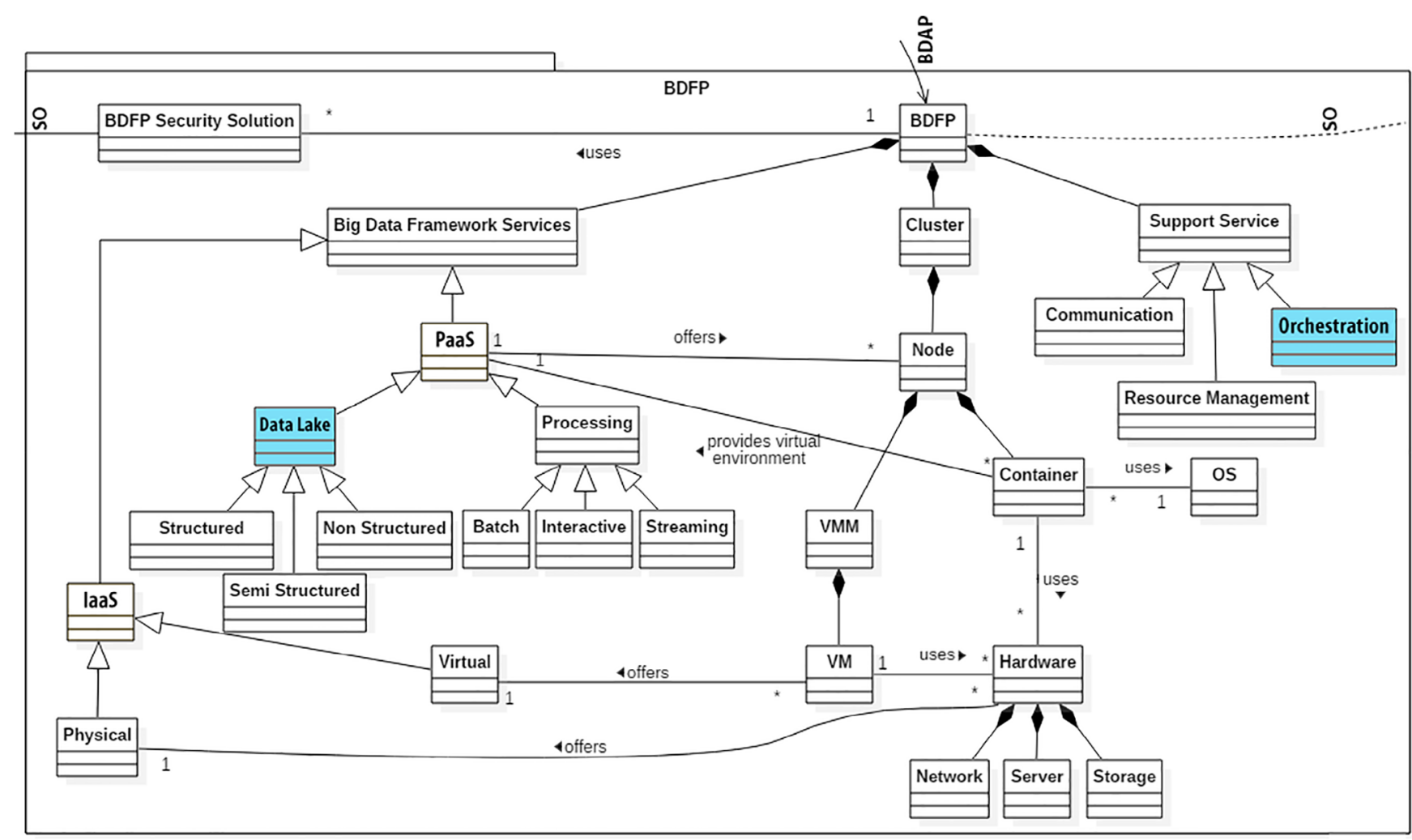

F I G U R E 7 Changes made to the Big Data framework provider (BDFP) component [Colour figure can be viewed at wileyonlinelibrary.com]

manner for the Big Data processes' orchestration. This type of service is widely used in such ecosystems: therefore, we added it to the SecRA. In the case study, this functionality is performed by the Azure Data Factory.

The storage system in a Big Data ecosystem is defined as the area where all the data that will be used later by the services of the system are stored. This definition includes raw data, the data prepared for future analysis, and the information resulting from analytical algorithms. In other words, several types of data coexist in the same storage. Consequently, such storage in Big Data environments is usually referred to as data lake; we followed the same convention in our architecture. Figure 7 illustrates the BDFP component and highlights the changes made in blue color.

In conclusion, we can affirm that our SecRA covers most of the elements present in the case study. It does so with small enhancements, thereby improving the case study's implementation. This is not surprising because the SecRA is based on standards that are very close to those of the industry. This relatedness to real-world environments renders our proposal useful to any user who wants to develop a Big Data system. Additional factors contributing to its usefulness are: it provides a global perspective of the system components and the main security artefacts to be considered.

\section{2 | Proposals for improvement of the case study}

In the course of this project, a series of meetings were held between the SecRA team and the team that implemented the Big Data ecosystem in the case study. During these meetings, the mapping between the elements of the SecRA and those implemented by the project was established and some security weaknesses of the implemented Big Data ecosystem were discovered. One of the identified problems was related to the management of permissions to Databricks through access tokens; these tokens grant temporary permissions to the users who normally cannot access the stored data. The problem arises because the generated tokens not only allow access to the data but also the creation of new tokens with different temporary restrictions.

Another problem that was identified is related to the landing area. The landing area layer of the system is used to temporarily store data before the application of security policies. This data must be accessible by only highly technical 
T A B L E 6 Security patterns that can be used to aid the implementation of the security requirements

\begin{tabular}{ll} 
Security Requirement & Security Patterns \\
RQ1. Data at rest must be encrypted & Symmetric encryption \\
RQ2. Data in transit must be encrypted & Secure channel \\
\hline RQ3. Allow users to bring their own key (BYOK) & Undeveloped pattern \\
\hline RQ4. Geographic allocation of the resources must be controlled & Undeveloped pattern \\
\hline RQ5. Protect personally identifiable information (PII) & $\begin{array}{c}\text { Multilevel security, Role-based access control, } \\
\text { Security logger/auditor, Access matrix }\end{array}$ \\
\hline RQ6. Control the management of rights (privileges) & Role-based access control, Authenticator \\
\hline RQ7. Vulnerability management & Undeveloped pattern \\
RQ8. Patch management & Undeveloped pattern \\
\hline RQ9. Provide activity log control & Security logger/auditor \\
\hline RQ10. Provide identity and access management (IAM) in all accounts & Credential, Role-based access control \\
\hline RQ11. Authenticate all connections & Distributed systems authenticator \\
\hline RQ12. Allow multifactor authentication (MFA) & Authenticator \\
\hline
\end{tabular}

profiles whose roles are not intended to analyze the data. We believe that although the implementation of the landing area is a good idea, it can be a gateway for malicious attacks. This is owing to the fact that highly sensitive data, although temporarily, is stored here, and it must be protected before it enters the system to avoid any threats to the system. A data leak in this layer could cause a disastrous impact on the company including fines for disclosure of confidential data. We recommend that the data stored in the Big Data ecosystem must be already processed to be able to comply with the regulations and security requirements. In other words, this processing should be done initially as indicated in the SecRA DP component.

We think that the security requirements in Table 2 are extremely simple and insufficiently detailed for an ecosystem as complex as the one implemented. This is a recurrent problem in Big Data ecosystems, where little emphasis is placed on the analysis and design phases of the system. Moreover, it is advisable to use an approach based on security-by-design, wherein security is considered since the early stages of project development. ${ }^{26}$ There is an absence of a systematic process that helps stakeholders to not only develop the requirements and policies (more specifically, the security ones) but also guide the development of the components of Big Data so that their implementation is aligned with the requirements and context of the environment. To mitigate these problems, we developed a process to implement secure Big Data that covers all phases till the implementation of the ecosystem; specifically, emphasizing the analysis and design phases. ${ }^{27}$

Furthermore, to aid the implementation of the requirements, we recommend the use of security best practices. Table 6 presents the security requirements of the case study and relates them to the security patterns that can be applied to solve them. The security patterns are defined in Reference 14 .

As shown in Table 6, not all security requirements have an existing specific security pattern, for example, we are not aware of a pattern that manages the patches of a tool (RQ8) or helps in the implementation of the BYOK policy (RQ3). In such cases, a specific security artifact must be implemented without the guidance of a pattern. However, there are patterns for most of the security requirements identified in the system. As an example, we discuss how the requirement on the management of system privileges (RQ6) can be implemented considering the different roles of the Big Data ecosystem (see Figure 8). Two patterns-role-based access control and an authenticator-can be used to implement this requirement.

As shown in Figure 8, there are four roles with different access privileges to the system components. The technician only has the right to read data in the landing area, whereas the data scientist has the right to read, modify, and delete data in the raw, common, and service layers - this is because of the fact that these roles are in charge of conducting analyses on the respective data. On the contrary, the reporter has the objective of formatting the information resulting from these analyses so that it can be understood by the top management; therefore, this role only has access to the service layer. Finally, the top management, the consumer of the data, only has the privilege of reading the resulting information (mainly reports and dashboards). All these roles must be authenticated prior to conducting any operation on the data. To this end, the roles must have an authentication proof that is created by an authentication service using authentication information. The users or roles must have previously assigned identity proofs. 


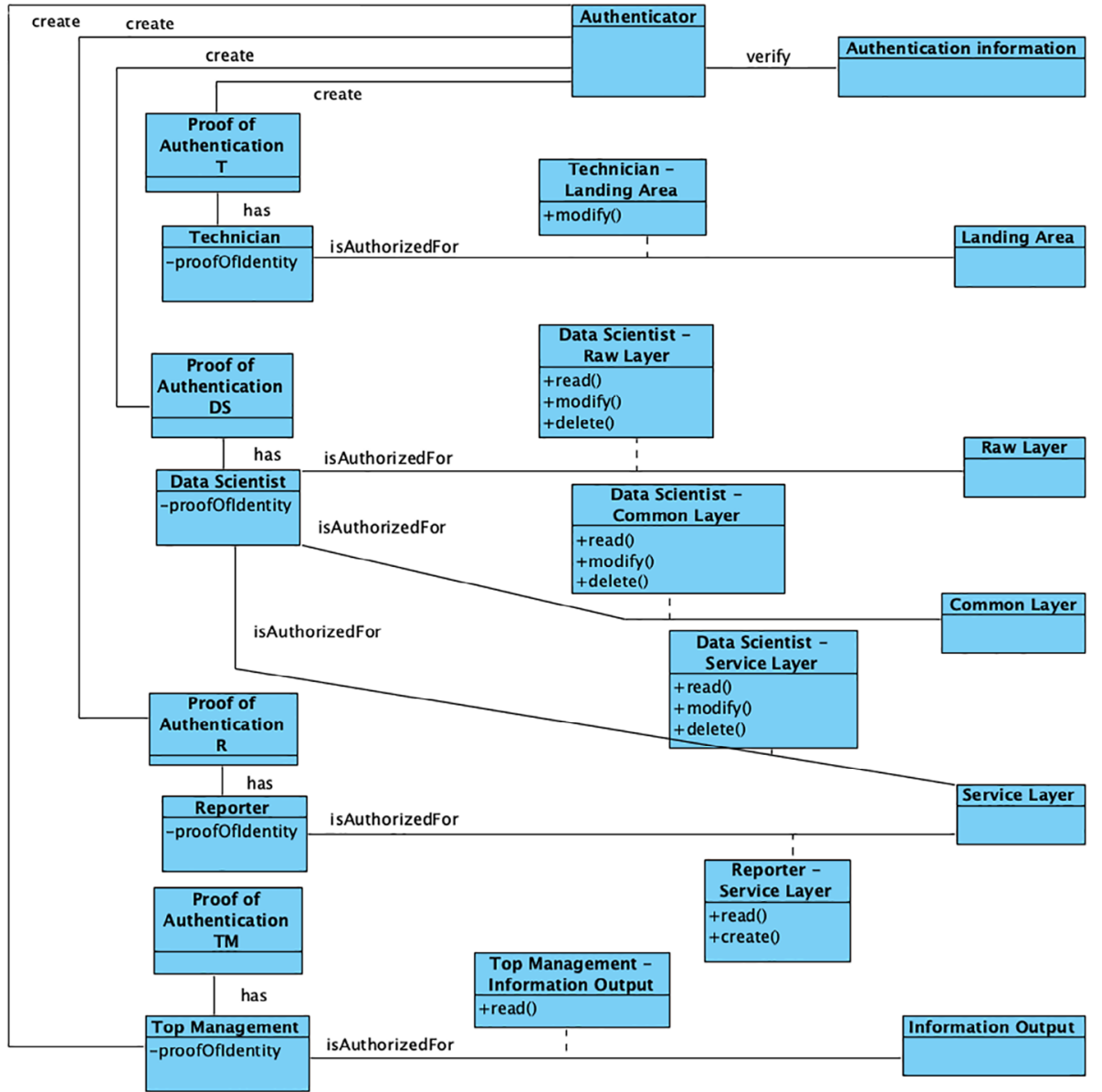

F I G U R E 8 Application of security pattern to aid the implementation of RQ6 [Colour figure can be viewed at wileyonlinelibrary.com]

Currently, the system has been launched as a "family and friends" version. At this stage, the environment can be accessed only by a reduced set of users with the aim of finding bugs and issues, before being launched to general users. The final release is scheduled for June 2020. The lessons learned in this exercise served to improve the security of the Big Data system and the implementation processes of such ecosystems by adding security patterns as a good practice.

\section{6 | CONCLUSIONS AND FUTURE WORK}

Implementing a Big Data ecosystem is not an easy task. Such systems are typically highly complex, with numerous software and hardware technologies working in collaboration. To address the problem of complexity, we proposed an SecRA based on the main industry standards. This SecRA aims to abstract the main components of Big Data and emphasize the need to consider security requirements from the early stages of development. As a first step in the validation of this architecture, we present a case study in this article that is based on a real-world implementation of a Big Data ecosystem in a banking environment by everis.

In this system, the security requirements are applied, thanks to the experience of everis developers who based their implementation strategy on a correct selection of the technologies to be used. Consequently, the main security requirements were met by configuring the different tools selected. However, there are other types of scenarios with different types of technologies and experience of the developers, wherein it is useful to have a standardized framework based on good practices to aid and guide the implementation of the required environment.

This article provides a set of lessons learned both to improve our SecRA and the implemented Big Data ecosystem. These improvements range from adding new elements to our SecRA to recommending best practices for the company, both for this Big Data ecosystem and future projects of the organization.

As future work, we will continue to collaborate with the everis company for further refinement and improvement of the security of both the current environment and the process of creating new Big Data ecosystems. In addition, 
we will continue with the validation of the SecRA by conducting new case studies in different contexts, for example, cyber-physical systems that use a Big Data ecosystem to analyze the actions to be performed by the actuators.

\section{ACKNOWLEDGEMENTS}

This work was funded by the ECLIPSE project (RTI2018-094283-B-C31 funded by “Ministerio de Economía y Competitividad and the Fondo Europeo de Desarrollo Regional FEDER"), the GENESIS project (SBPLY-17-180501-000202 funded by "Consejería de Educación, Cultura y Deportes de la Dirección General de Universidades, Investigación e Innovación de la JCCM"), and the Programa Operativo Regional FEDER 2014/2020.

The authors would like to thank everis, an NTT Company (https://www.everis.com/global/en) for their continued support.

\section{ORCID}

Julio Moreno (D) https://orcid.org/0000-0001-9974-1199

\section{REFERENCES}

1. Akoka J, Comyn-Wattiau I, Laoufi N. Research on big data-a systematic mapping study. Comput Stand Interfac. 2017;54:105-115. https:// doi.org/10.1016/j.csi.2017.01.004.

2. Columbus L. 10 charts that will change your perspective of big data's growth. https://www.forbes.com/sites/louiscolumbus/2018/05/23/ 10-charts-that-will-change-your-perspective-of-big-datas-growth/. Accessed May 13, 2019.

3. Alic AS, Almeida J, Aloisio G, et al. BIGSEA: a big data analytics platform for public transportation information. Futur Gener Comput Syst. 2019;96:243-269. https://doi.org/10.1016/j.future.2019.02.011.

4. Bhuiyan MZA, Zaman A, Wang T, Wang G, Tao H, Hassan MM. Blockchain and big data to transform the healthcare. Paper presented at: Proceedings of the International Conference on Data Processing and Applications; ACM; New York, NY; 2018:62-68. https://doi.org/10. $1145 / 3224207.3224220$.

5. ur Rehman MH, Yaqoob I, Salah K, Imran M, Jayaraman PP, Perera C. The role of big data analytics in industrial Internet of Things. Futur Gener Comput Syst. 2019;99:247-259. https://doi.org/10.1016/j.future.2019.04.020.

6. Demchenko Y, De Laat C, Membrey P. Defining architecture components of the Big Data Ecosystem. Paper presented at: 2014 International Conference on Collaboration Technologies and Systems (CTS); Minneapolis, MN; IEEE; 2014:104-112.

7. Garlan D, Bachmann F, Ivers J, et al. Documenting Software Architectures: Views and Beyond. Boston, Massachusetts: Addison-Wesley Professional; 2010.

8. Fernandez EB, Monge R, Hashizume K. Building a security reference architecture for cloud systems. Requir Eng. 2016;21:225-249. https:// doi.org/10.1007/s00766-014-0218-7.

9. Moreno J, Serrano MA, Fernandez-Medina E, Fernandez EB. Towards a security reference architecture for big data. Paper presented at: the DOLAP Workshop Colocated With EDBT/ICDT Conference. 2018.

10. Avgeriou P. Describing, instantiating and evaluating a reference architecture: a case study. Default J. 2003;342:1-24.

11. ISO/IEC CD 20547-3. Information technology—Big data reference architecture-Part 3: Reference architecture. https://www.iso.org/ standard/71277.html?browse=tc. Accessed January 15, 2019.

12. NBD-WG. NIST: NIST Big Data Reference Architecture. https://bigdatawg.nist.gov/_uploadfiles/M0639_v1_9796711131.docx. Accessed January 10, 2019.

13. NIST Big Data Public Working Group. NIST Big Data Interoperability Framework. Vol 4. Secur Priv. https://nvlpubs.nist.gov/nistpubs/ SpecialPublications/NIST.SP.1500-4r1.pdf. Accessed September 12, 2018.

14. Fernandez EB. Security Patterns in Practice: Designing Secure Architectures Using Software Patterns. Hoboken, NJ: John Wiley \& Sons; 2013.

15. Steel C, Nagappan R, Lai R. The alchemy of security design methodology, patterns, and reality checks. Core Security Patterns: Best Practices and Strategies for J2EE, Web Services, and Identity Management. Upper Saddle River, New Jersey: Prentice Hall; 2005.

16. Pääkkönen P, Pakkala D. Reference architecture and classification of technologies, products and services for big data systems. Big Data Res. 2015;2:166-186.

17. Nadal S, Herrero V, Romero O, et al. A software reference architecture for semantic-aware big data systems. Inf Softw Technol. 2017;90:75-92. https://doi.org/10.1016/j.infsof.2017.06.001.

18. Marz N, Warren J. Big Data: Principles and Best Practices of Scalable Real-Time Data Systems. New York, NY: Manning Publications Co; 2015.

19. Darwish TSJ, Bakar KA. Fog based intelligent transportation big data analytics in the internet of vehicles environment: motivations, architecture, challenges, and critical issues. IEEE Access. 2018;6:15679-15701. https://doi.org/10.1109/ACCESS.2018.2815989.

20. Psomakelis E, Tserpes K, Zissis D, Anagnostopoulos D, Varvarigou T. Context agnostic trajectory prediction based on $\lambda$-architecture. Futur Gener Comput Syst. 2019. https://doi.org/10.1016/j.future.2019.09.046.

21. Klein J, Buglak R, Blockow D, Wuttke T, Cooper B. A reference architecture for big data systems in the national security domain. Paper presented at: Proceedings of the 2nd International Workshop on BIG Data Software Engineering; ACM; Austin, TX; 2016:51-57.

22. Mistrik I, Bahsoon R, Ali N, Heisel M, Maxim B. Software Architecture for Big Data and the Cloud. Elsevier; 2017. https://doi.org/10.1016/ C2015-0-01431-9. 
23. Zhang X, Ming X, Yin D. Reference architecture of common service platform for industrial big data (I-BD) based on multi-party co-construction. Int J Adv Manuf Technol. 2019;105:1949-1965. https://doi.org/10.1007/s00170-019-04374-X.

24. Coghlan D. Doing Action Research in Your Own Organization. London: SAGE Publications Limited; 2019.

25. Kock N, Lau F. Information systems action research: serving two demanding masters. Inf Technol People. 2001;14. https://doi.org/10.1108/ itp.2001.16114aaa.001.

26. Casola V, De Benedictis A, Rak M, Rios E. Security-by-design in clouds: a security-SLA driven methodology to build secure cloud applications. Procedia Comput Sci. 2016;97:53-62. https://doi.org/10.1016/j.procs.2016.08.280.

27. Moreno J, Fernandez EB, Serrano MA, Fernández-Medina E. Secure development of big data ecosystems. IEEE Access. 2019;7:96604-96619. https://doi.org/10.1109/ACCESS.2019.2929330.

How to cite this article: Moreno J, Gómez J, Serrano MA, B. Fernandez E, Fernández-Medina E. Application of security reference architecture to Big Data ecosystems in an industrial scenario. Softw: Pract Exper.

2020;50:1520-1538. https://doi.org/10.1002/spe.2829 